\title{
Unravelling genome dynamics in Arabidopsis synthetic auto and allopolyploid species
}

\author{
M. BENTO, D. TOMÁS, W. VIEGAS, and M. SILVA* \\ Linking Landscape, Environment, Agriculture and Food (LEAF), Instituto Superior de Agronomia, \\ Universidade de Lisboa, Tapada da Ajuda 1349-017 Lisboa, Portugal
}

\begin{abstract}
Polyploidization is a major genome modification that results in plant species with multiple chromosome sets. Parental genome adjustment to co-habit a new nuclear environment results in additional innovation outcomes. We intended to assess genomic changes in polyploid model species with small genomes using inter retrotransposons amplified polymorphism (IRAP) and retrotransposon microsatellite amplified polymorphism (REMAP). Comparative analysis among diploid and autotetraploid A. thaliana and A. suecica lines with their parental lines revealed a marginal fraction of novel bands in both polyploids, and a vast loss of parental bands in allopolyploids. Sequence analysis of some remodelled bands shows that $A$. suecica parental band losses resulted mainly from sequence changes restricted to primer domains. Moreover, in A. suecica, both parental genomes presented rearrangement frequencies proportional to their sizes. Overall rates of genomic remodelling events detected in A. suecica were similar to those observed in species with a large genome supporting the role of retrotransposons and microsatellite sequences in the evolution of most allopolyploids.
\end{abstract}

Additional key words: microsatellites, polyploidization, retrotransposons, sequence rearrangement.

\section{Introduction}

Recent plant genomic studies have evidenced the crucial relevance of labile repetitive sequences like retrotransposons in plant evolution through polyploidization. Due to the huge amount of genetic data available from species with small genomes like Arabidopsis sp., the study of repetitive genome fraction involvement in polyploid induced remodelling events may be particularly revealing in this genus. In fact polyploidy, which result from the fusion of two or more complete genomes into the same nucleus, is present in the evolution route of most flowering plants with both large and small genome (Jiao et al. 2011). Distinct types of polyploids can arise depending on the origins of genomes involved: autopolyploids originated from genome doubling within the same species and allopolyploids formed by the combination of two or more genomes from distinct but usually close species (Ma and Gustafson 2008).

The impact of polyploidy on genome organization and function (reviewed in Ainouche 2010 and Stöck and Lamatsch 2013) was extensively pursued using different approaches, and it is presently well established that polyploidy induces innumerable genetic and epigenetic modifications on parental genomes (Feldman and Levy 2009, Jones and Hegarty 2009). Genomic studies using amplified fragment length polymorphism (AFLP), although having the advantage of full genome coverage, usually reveal lower levels of rearrangements associated with polyploidization when compared with techniques targeting major types of repetitive sequences widely present in plant genomes like retrotransposons and microsatellites (Ma et al. 2004, Madlung et al. 2005, Bento et al. 2008, 2013). From these techniques, inter retrotransposons amplified polymorphism (IRAP) and retrotransposon microsatellite amplified polymorphism (REMAP) have recently demonstrated the marked importance of retrotransposons as a particularly dynamic

Submitted 24 November 2014, last revision 31 March 2015, accepted 1 April 2015.

Abbreviations: AFLP - amplified fragment length polymorphism; IRAP - inter retrotransposons amplified polymorphism; LTR - long terminal repeats; REMAP - retrotransposon microsatellite amplified polymorphism; SSR - simple sequence repeats.

Acknowledgements: M. Bento was funded by a FCT (Fundação para a Ciência e a Tecnologia, Portugal) postdoctoral grant (SFRH/BPD/80550/2011), Diana Tomás was funded by a FCT doctoral scholarship (SFRH/BD/93156/2013), Manuela Silva by the FCT Investigator Programme (IF/00834/2014), and the research work was financed by FCT LEAF Unit (UID/AGR/04129/2013).

* Corresponding author, fax: (+351) 213653238, e-mail address: manuelasilva@isa.ulisboa.pt 
fraction in processes underlying polyploid genome adjustments (Bento et al. 2008, 2013). Furthermore, the evaluation of microsatellite dynamics in Brassica, Arabidopsis, and other angiosperms indicate that polyploidization may induce a slight increase of microsatellite frequency in coding regions although associated with a significant decrease in whole genome/non-coding sequences (Shi et al. 2013). These evidences reinforce the current well-established notion that genome size changes in allopolyploids are correlated with changes on repetitive genome fractions (RennyByfield et al. 2013).

Most studies targeting genomic rearrangements in plant allopolyploids were pursued in species with medium and large genomes, such as octoploid triticale $(1 \mathrm{C}=25404 \mathrm{Mb}$; monoploid genome size $1 \mathrm{Cx}=6351 \mathrm{Mb})$, hexaploid Triticum aestivum $(1 \mathrm{C}=16944 \mathrm{Mb}$; $1 \mathrm{Cx}=5648 \mathrm{Mb})$, tetraploid Nicotiana tabacum $(1 \mathrm{C}=5061 \mathrm{Mb} ; 1 \mathrm{Cx}=2530 \mathrm{Mb})$, dodecaploid Spartina anglica $(1 \mathrm{C}=5330 \mathrm{Mb} ; 1 \mathrm{Cx}=888 \mathrm{Mb})$, and tetraploid Cucumis hytivus (diploid parental line C. sativus $1 \mathrm{C}=880 \mathrm{Mb}$ ) (Bento et al. 2008, Feldman and Levy 2009, Jiang et al. 2009, Parisod et al. 2009, Petit et al. 2010). Deoxyribonucleic acid C-values were obtained from the Plant DNA C-values database (Bennett and Leitch 2010; http://www.kew.org/cvalues/) with an estimated repeat content of $>80 \%, \sim 80 \%, \sim 70 \%, \sim 30 \%$, and $\sim 24 \%$, respectively (Dvořák 2009, Huang et al. 2009, Brenchley et al. 2012, Ferreira-de-Carvalho et al. 2013, Sierro et al.
2013). In species with small genomes, such as Arabidopsis with a lower repetitive sequences fraction $(\sim 14 \%)$ (AGI 2000), the assessment of alterations induced by polyploidy in such a genome portion was meagerly studied. Due to vast genetic and genomic resources available (http://www.arabidopsis.org/), Arabidopsis polyploid species constitute a particularly attractive model to such studies (reviewed in Bomblies and Madlung 2014). Previous studies of newly formed autotetraploid lines of $A$. thaliana revealed minor genomic changes induced by polyploidy (Santos et al. 2003, Madlung et al. 2005, Ozkan et al. 2006), though epigenetic modifications were reported ( $\mathrm{Yu}$ et al. 2010). A. suecica $(2 \mathrm{n}=4 \mathrm{x}=26)$, the natural allopolyploid between $A$. thaliana and $A$. arenosa, can be synthetically produced by crossing $A$. thaliana $(2 \mathrm{n}=4 \mathrm{x}=20)$ and $A$. arenosa $(2 \mathrm{n}=4 \mathrm{x}=32)$ autotetraploid lines (Madlung et al. 2005). The analysis of Arabidopsis allopolyploids revealed both genetic (Madlung et al. 2005, Pecinka et al. 2011, Henry et al. 2014) and epigenetic modifications (Hazzouri et al. 2008, Beaulieu et al. 2009, Yu et al. 2010). Our previous results obtained using IRAP methodology also demonstrated the occurrence of genomic rearrangements induced by allopolyploidization in A. suecica (Bento et al. 2013). In this work, we intended to understand the implication of retrotransposons and also microsatellite related sequences in genetic modifications induced by auto and allopolyploidization in the Arabidopsis species.

\section{Materials and methods}

The following plants were used: diploid Arabidopsis thaliana L. (cv. Landsberg erecta, Ler- $0 ; 2 n=2 x=10$, NASC stock number NW20) and the equivalent autotetraploid line (LC612; $2 \mathrm{n}=4 \mathrm{x}=20, N A S C$ stock No. $\mathrm{N} 3900) ; A$. arenosa L. (CARE-1; $2 \mathrm{n}=4 \mathrm{x}=32, N A S C$ stock No. N3901) and the newly synthesized allopolyploid A. suecica Fr. line (Ler-0 x CARE-1; 2n=4x=26; F4 generation, NASC stock No. N3899). The $A$. thaliana autotetraploid and $A$. arenosa lines used are the exact parents of the referred newly synthesized allopolyploid. All seeds were obtained from the NASC-European Arabidopsis Stock Centre and germinated and grown in controlled conditions at a $16-\mathrm{h}$ photoperiod, an irradiance of $150 \mu \mathrm{mol} \mathrm{m} \mathrm{m}^{-2} \mathrm{~s}^{-1}$, day/night temperatures of $22 / 20{ }^{\circ} \mathrm{C}$, and a relative humidity of $70 \%$. At least four distinct plants from each genotype were analyzed. Genomic DNA was isolated from fresh young leaves of eight-week-old plants using a modified cetyltrimethylammonium bromide (CTAB) method (Saghai-Maroof et al. 1984).

The used PCR-based methodologies included IRAP and REMAP. The IRAP amplify sequences between retrotransposons using primers pointing outward from the long terminal repeats (LTR) end. The REMAP uses the

same LTR primers combined with primers matching simple sequence repeats (SSR) or microsatellite motifs with one or more non-SSR anchor nucleotides thus amplifying segments between LTRs, between LTR and SSR, or between nearby microsatellites (Kalendar and Schulman 2006). The IRAP was prepared using primers for LTR regions of distinct A. thaliana retrotransposons including Metaviridae (Tat1, Athila4-6, and RomaniaT5) as well as Pseudoviridae (Endovir1-1). The REMAP was done using an Endovir1-1 LTR primer and an anchored microsatellite primer (AAG)6C. All primers used are listed in Table 1. The PCR products were run on $1.7 \%$ $(\mathrm{m} / \mathrm{v})$ agarose gels, detected with ethidium bromide staining, and photographed using BioRad (Hercules, USA) GEL DOC 2000 (Tables 2 and 3). Reproducible results presented were accurately observed in at least three replicates of each IRAP and REMAP experiment. Results obtained in A. suecica with the Tat 1 and Athila 4 - 6 primers were previously presented in Bento et al. (2013). Differences observed between diploid and tetraploid $A$. thaliana profiles unraveled autopolyploidization induced genomic changes. Constancy observed in IRAP/REMAP banding profiles on all 
A. thaliana and A. arenosa plants analyzed allowed an accurate comparison of the neosynthetized allopolyploid with both parental lines. In $A$. suecica, genomic rearrangements were disclosed by differences between its banding profile and the addition of the parental lines banding profiles. Moreover, to confirm that polyploid lost bands did not result from competition between priming sites of parental genomes, a mix of the parental DNAs was used (results not shown) as previously described in detail (Bento et al. 2008).

Rearranged sequences were isolated, purified, cloned, and finally sequenced following procedures described in Rocheta et al. (2007). The sequences obtained were used for BLASTN (algorithm parameters set for default values) on the NCBI (the National Center for Biotechnology Information), TAIR (the Arabidopsis Information Resource), and PlantSat (repetitive plant sequences) databases. Additionally, primers were designed to amplify internal fragments of rearranged sequences (Table 1). The PCR amplifications and analysis of internal sequences were performed as described in Bento et al. (2010).

Table 1. Primers used for PCR analyses.

\begin{tabular}{llll}
\hline Retroelement & GeneBank acc. No. & Primer & Superfamily \\
\hline Tat 1 & AF056631 & 5'-TCCTCGTAAAACAACCACAAG & Gypsy-like \\
Athila4-6 & AF296831 & 5'-AAATGGATGCTCAAAACATGC & Gypsy-like \\
RomaniaT5 & AF073829 & 5'-GGTTTCTGAGGATGGGTTAG & Gypsy-like \\
Endovir1-1 & AY016208 & 5'-CCAAATGAACGAATGACGTG & Copia-like \\
Microsatellite (AAG) 6 C & & 5'-AAGAAGAAGAAGAAGAAGC & \\
\hline
\end{tabular}

\begin{tabular}{lll}
\hline Internal segments & Forward & Reverse \\
\hline Romania_aa951[1] & 5'-TGCCTGCTAGGTTATGTTTC & 5'-CATGCATAAAGGGAAATAGC \\
Romania_aa951[2] & 5'-ATTCTCTGTACTTGCTACTTGC & 5'-GCGCCAAATTGATACTAGG \\
Romania_aa1357[2] & 5'-GTGTGGTCGAGTATCAGGTC & 5'-GATTCGTAATGCCTGTTAGG \\
Romania_aa1357[1] & 5'-TCAAGATGCAATCAGATGTC & 5'-TAACAAACTGTGCAGTCGAG \\
End-AAG_as552[2] & 5'-ATTCTAGCCATTGTTTCGTC & 5'-GTGGGTGTTGTGATTACCC \\
End-AAG_as552[1] & 5'-GATTGGTGGCAGGGATAC & 5'-CTTGTTTGTCCTCCTTTTTG \\
\hline
\end{tabular}

\section{Results}

To assess genomic rearrangements induced by polyploidization on Arabidopsis autopolyploids and allopolyploids, IRAP and REMAP experiments with five primer combinations were performed including four primers designed for LTR regions of Arabidopsis retrotransposons (Tat1, Athila4-6, RomaniaT5, and Endovir1-1) and one anchored microsatellite primer [(AAG)6C] (Table 1). Unique and reproducible banding profiles from diploid and tetraploid $A$. thaliana, $A$. arenosa, and $A$. suecica were consistently obtained in all PCR replicates from at least four distinct plants of each species and primer combination. Thus, a parental comparative evaluation of auto and allopolyploid lines was performed to assess the impact of polyploidization in retrotransposon and microsatellite flanking sequences (Fig. 1).

To unravel potential genomic rearrangements associated with autopolyploidization banding profiles of original diploid $A$. thaliana and those of the autotetraploid line were compared (Table 2). Additionally, a new band of $\sim 1700 \mathrm{bp}$, absent in the diploid parental line, was unravelled in the RomaniaT5 IRAP profile of the autotetraploid (Fig. 1). Thus, a reduced overall frequency of $1.7 \%$ novel bands (1/58) was

Table 2. The IRAP and REMAP analyses of autotetraploid $A$. thaliana.

\begin{tabular}{llllllll}
\hline Ploidy & Bands & $\begin{array}{l}\text { IRAP } \\
\text { Tat1 }\end{array}$ & Athila4-6 & RomaniaT5 & Endovir1-1 & $\begin{array}{l}\text { REMAP } \\
\text { Endovir1-1/(AAG)6C }\end{array}$ & Total \\
\hline Diploid & observed bands & 8 & 11 & 7 & 17 & 15 & 58 \\
Tetraploid & conserved bands & 8 & 11 & 7 & 17 & 15 & 58 \\
& novel bands & 0 & 0 & 1 & 0 & 0 & 1 \\
\hline
\end{tabular}




\section{BENTO et al.}

Table 3. The IRAP and REMAP analyses of synthetic allopolyploid A. suecica and parental lines (* - results previously published in Bento et al. (2013); [i] - monomorphic bands: common to both A. suecica progenitors; [ii] - polymorphic bands: observed in only one A. suecica progenitor; [iii] - A. suecica expected bands: the sum of parental bands observed; [iv] - A. suecica observed bands: bands observed in the allopolyploid; [v] - A. suecica rearranged bands: parental bands absent and novel bands observed only in the polyploidy).

\begin{tabular}{|c|c|c|c|c|c|c|}
\hline & $\begin{array}{l}\text { IRAP } \\
\text { Tat }{ }^{*}\end{array}$ & Athila4-6* & RomaniaT5 & Endovir $1-1$ & $\begin{array}{l}\text { REMAP } \\
\text { Endovir1-1/(AAG)6C }\end{array}$ & Total \\
\hline A. thaliana tetraploid & 8 & 11 & 8 & 17 & 15 & 59 \\
\hline A. arenosa & 12 & 8 & 10 & 12 & 16 & 58 \\
\hline \multicolumn{7}{|l|}{ A. thatiana vs. $A$. arenosa } \\
\hline monomorphic bands [i] & 2 & 0 & 2 & 8 & 7 & 19 \\
\hline polymorphic bands [ii] & 16 & 19 & 14 & 13 & 17 & 79 \\
\hline \multicolumn{7}{|l|}{ A. suecica } \\
\hline expected [iii] & 18 & 19 & 16 & 21 & 24 & 98 \\
\hline observed bands [iv] & 10 & 16 & 7 & 13 & 19 & 65 \\
\hline Rearranged bands [v] & 10 & 3 & 9 & 8 & 9 & 39 \\
\hline lost from $A$. thaliana & 3 & 1 & 2 & 6 & 0 & 12 \\
\hline lost from $A$. arenosa & 6 & 2 & 7 & 2 & 7 & 24 \\
\hline novel bands & 1 & 0 & 0 & 0 & 2 & 3 \\
\hline
\end{tabular}

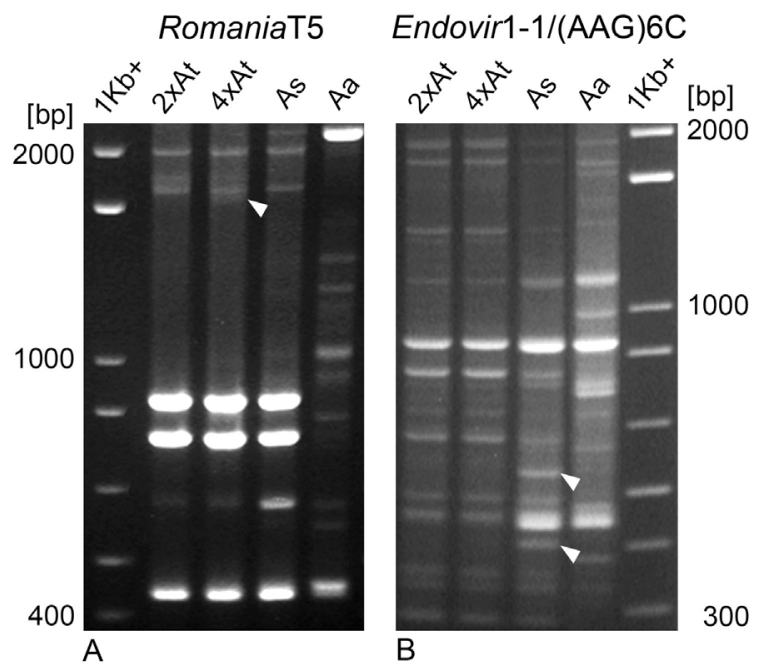

Fig. 1. The IRAP banding profiles obtained with primers for RomaniaT5 and REMAP banding profiles obtained with primers for Endovir1-1/(AAG)6C of $A$. thaliana Ler-0 $2 \mathrm{n}=2 \mathrm{x}=10$ (2xAt), A. thaliana LC612 $2 \mathrm{n}=4 \mathrm{x}=20$ (4×At), A. suecica synthetic allopolyploid $2 \mathrm{n}=4 \mathrm{x}=26$ (As), and $A$. arenosa CARE-1 $2 \mathrm{n}=4 \mathrm{x}=32$ (Aa). Arrows indicate novel bands observed in the autopolyploid $(A)$ and in the allopolyploid $(B)$ lines. Molecular mass marker is marked as $1 \mathrm{~Kb}+$.

detected in the autopolyploid line. The same comparative analysis of allopolyploid $A$. suecica in relation to the tetraploid $A$. thaliana and A. arenosa lines (Table 3) revealed one novel band with Tat 1 IRAP and two with Endovir1-1/(AAG)6C REMAP corresponding to a $4.6 \%$ (3/65) frequency.

Two novel bands uncovered in $A$. suecica with REMAP Endovir1-1/(AAG)6C were isolated, purified, and cloned for sequencing, and both sequences revealed to be allocated between microsatellite loci. Moreover, their BLASTN analysis on the NCBI, TAIR, and PlantSat databases (Fig. 2 and Table 1 Suppl.) unravelled homology with $A$. thaliana coding sequences mapped on chromosomes 3 and 5, respectively. End-AAG_as415
(415 bp, acc. No. KJ371929) presented a $78 \%$ similarity and a $96 \%$ coverage with an $A$. thaliana coding region corresponding to a gyp $1 p$ superfamily protein. Again, End-AAG_as552 sequence (552 bp, acc. No. KJ371930) showed a $77 \%$ homology with the coding region of a RING-H2 finger protein isolated from the same species but only with a $36 \%$ coverage since a $1-355$ bp region did not produce any significant alignment with the A. thaliana published sequence. However, a 1 - 181 bp domain revealed a $92 \%$ homology with an unknown sequence of one Arabidopsis halleri BAC library (acc. No. FO203486.1).

To better understand the origin of the A. suecica novel sequence, we designed primers to amplify partial internal 
fragments of the End-AAG_as552 sequence (primer details in Table 1 and a schematic representation in Fig. 2). The obtained results (Fig. 3A) confirmed the amplification of three targeted internal fragments of A. suecica with the expected sizes (Fig. 2 and Table 2 Suppl.): End-AAG_as552_[1] (247 bp); End-
AAG_as552_[2] (268 bp); and End-AAG_as552_[1-2] $(408 \overline{\mathrm{b} p})$ which resulted from the amplification of the forward primer [1] with the reverse primer [2]. In both the parental species, no amplification products were obtained with internal primers confirming the novelty of the End-AAG_as552 sequence identified in $A$. suecica.
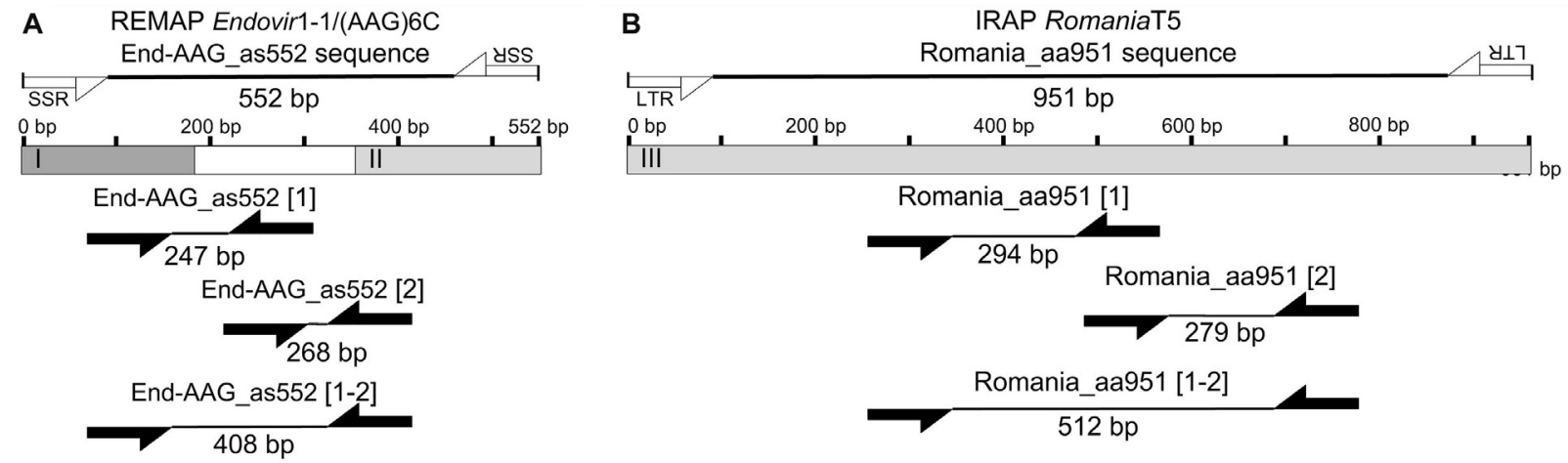

C

IRAP RomaniaT5

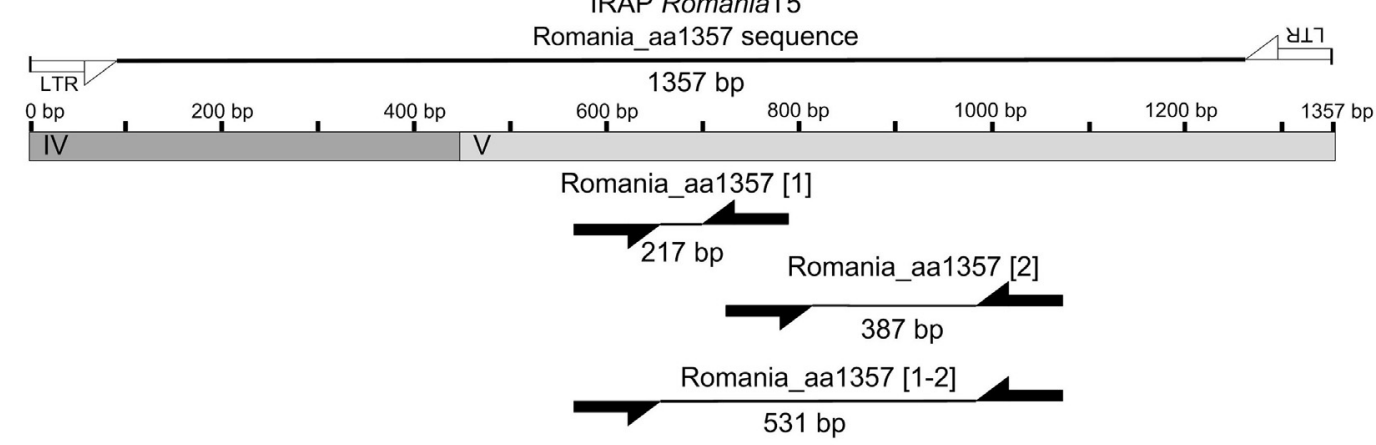

Fig. 2. A. suecica rearranged sequence characterization (alignments detailed in Table 1 Suppl.) and primers designed to amplify internal segments (primer details in Tables 1 and 2 Suppl.). Primers used in IRAP and REMAP initial experiments are represented in white and regions revealing similarity with published sequences (the NCBI, TAIR, and PlantSat databases) are indicated (I, II, III, IV, and V). Primers designed to amplify internal segments are represented in black and the dimensions of expected internal fragments are also illustrated. $A$ - an REMAP End-AAG_as552 A. suecica novel band: I - a 1 to 181 bp region similar to A. halleri BAC; II - a 356 to 552 bp region similar to A. thaliana RING-H2 finger protein ATL74 coding region; expected internal segments: End-AAG_as552 [1], End-AAG_as552 [2], and End-AAG_as552 [1-2]. B - an IRAP Romania_aa951 sequence from A. arenosa absent in $\bar{A}$. suecica: III - the total span of the sequence similar to the partial region of a gypsy-like retrotransposon family (Athila); LTR - expected internal segments: Romania_aa951 [1], Romania_aa951 [2], and Romania_aa951 [1-2]. C - an IRAP Romania_aa1357 sequence from $A$. arenosa absent in $A$. suecica: IV - a 1-446pb region composed by $\sim 2.5$ copies of the 180 bp centromeric repeats of $A$. arenosa; V - a 447-1357 bp region similar to A. thaliana Athila family retrotransposons; expected internal segments: Romania_aa1357 [1], Romania_aa1357 [2], and Romania_aa1357 [1-2].

A. suecica progenitor lines (tetraploid $A$. thaliana and A. arenosa) had markedly distinct profiles with a total of 59 and 58 bands, respectively, comprehending mainly species-specific bands (polymorphic bands) and a minor fraction (19/98 19\%) of common bands (monomorphic bands) (Table 3). Thus, absolutely additive banding profiles were expected to render 98 distinctive bands in the allopolyploid, markedly contrasting with the total number of 65 bands detected in the neosynthetized A. suecica. The high frequency $(36 / 98 \sim 37 \%)$ of bands absent in $A$. suecica comprehended $67 \%$ (24/36) of $A$. arenosa-specific and $33 \%(12 / 36)$ of $A$. thalianaspecific bands.
Sequence analysis of parental bands undetected in A. suecica through IRAP and REMAP (Table 1 Suppl.) included one $A$. thaliana-origin band and five $A$. arenosaorigin bands, namely: 1) Tat_at944 (A. thaliana Tat 1 IRAP, 944 bp, acc. No. KJ371934); 2) Tat_aa740 (A. arenosa Tat 1 IRAP, 740 bp, acc. No. KJ371931); 3) Endovir_aa929 (A. arenosa Endovir1-1 IRAP, 929 bp, acc. No. KJ371933); 4) End-AAG_aa387 (A. arenosa Endovir1-1/(AAG)6C REMAP, 387 bp, acc. No. KJ371928); 5) Romania aa951 (A. arenosa RomaniaT5 IRAP, 951 bp, acc. No. KJ371932); and 6) Romania_aa1357 (A. arenosa RomaniaT5 IRAP, 1357 bp, acc. No. KJ371935). The BLAST alignments of 
A. suecica lost sequences revealed a significant homology with both repetitive and coding sequences. Four restructured sequences presented very significant alignments with protein coding regions of the $A$. thaliana genome mapped on distinct chromosomes: Tat_at944 chromosome 1; Tat aa740 - chromosome 3; Endovir_aa929 - chromosome 3; and End-AAG_aa387 chromosome 4. From them, the REMAP End-AAG_aa387 sequence was proved to be amplified through the (AAG)6C anchored primer as described for the A. suecica novel bands obtained through the same REMAP experiment. On the other hand, Romania_aa951 and Romania_aa1357 sequences produced significant alignments with all $A$. thaliana centromeric regions. Romania_aa951 corresponded mainly to a partial region of the LTR of a gypsy-like retrotransposon family (Athila), and the Romania_aa1357 initial part (1 - 446 pb) was composed by $\sim 2.5$ units of 180 bp centromeric repeats whereas the remaining part corresponded also to
Athila-like retrotransposon sequences.

To deeper assess the involvement of retrotransposon related sequences in parental genome adjustment in the allopolyploid line, internal primers were designed for $A$. arenosa remodelled sequences Romania_aa951 and Romania aa1357 (primers in Table 1 and a schematic representation in Fig. 2). The results obtained in $A$. arenosa confirmed the amplification of three Romania_aa951 internal fragments with expected sizes (Table $\overline{2}$ Suppl.): Romania_aa951_[1] (294 bp); Romania_aa951_[2] (279 bp); and Romania_aa951_[1-2] $(512 \mathrm{bp})$, which resulted from the amplification of the forward primer [1] with the reverse primer [2]. Contrary to the original Romania_aa951 band, the three predictable internal segments were also detected in the $A$. suecica allopolyploid. Romania aa951 segments [1] and [2] were absent in A. thaliana di- and tetra-ploid lines (Fig. $3 B$ ) although the primers for segment [1-2] amplified two bands with $\sim 400$ and $\sim 550$ bp in both $A$. thaliana lines.

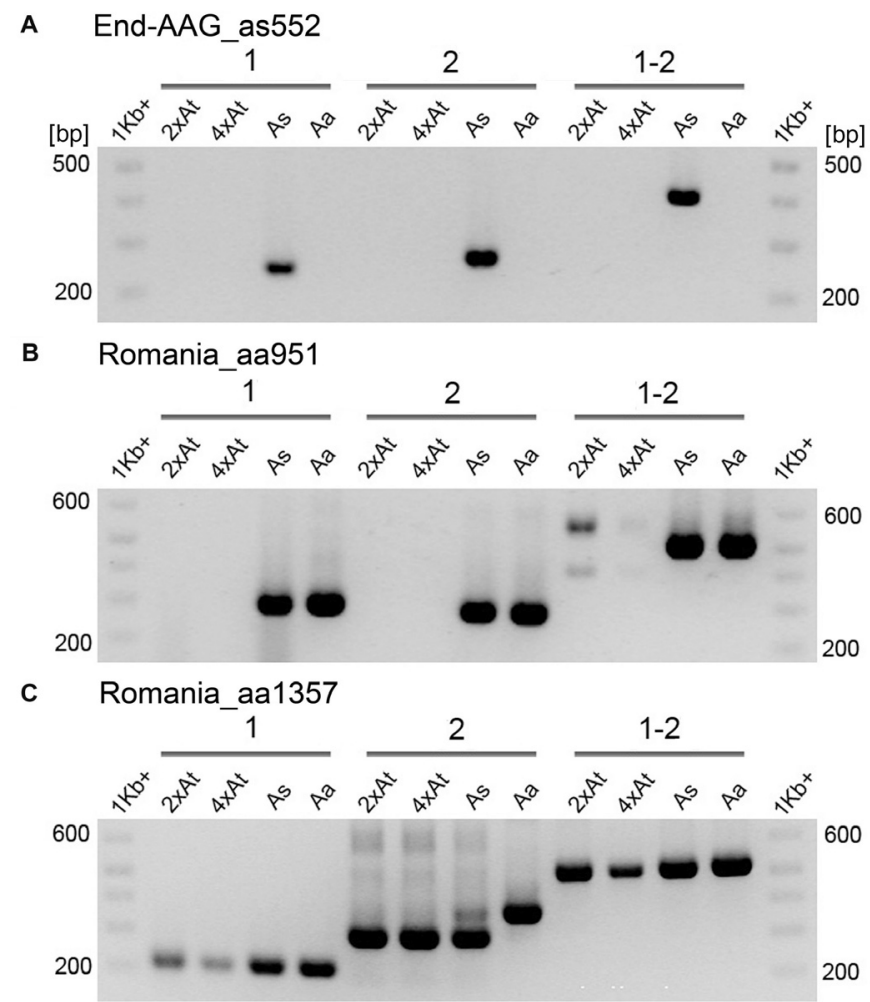

Fig. 3. The PCR banding profiles obtained with primers designed to amplify internal segments of rearranged sequences identified in A. suecica: $A$ - End-AAG_as552 [1], [2], and [1-2], $B$ - Romania_aa951 [1], [2], and [1-2], $C$ - Romania_aa1357[1], [2], and [1-2]. A. thaliana $(2 \times \mathrm{At})$, A. thaliana $(4 \times \mathrm{At})$, A. suecica synthetic allopolyploid (As), and A. arenosa (Aa). The details of all primers used are presented in Table 1 and Table 2 Suppl. Molecular mass marker is marked as $1 \mathrm{~Kb}^{+}$.

Concerning Romania_aa1357, the designed primers targeted only a domain similar to Athila retrotransposons (Fig. 2) and the results obtained in A. arenosa confirmed the amplification of three fragments with expected sizes (Table 2 Suppl.): Romania_aa1357_[1] (217 bp); Romania_aa1357_[2] (387 bp); and Romania_aa1357_[1-2]
(531 bp), which resulted from the amplification of the forward primer [1] with the reverse primer [2]. As observed for Romania_aa951, all Romania_aa1357 internal segments were found in the A. suecica allopolyploid (Fig. 3C). Moreover, the internal segments Romania_aa1357_[1] and Romania_aa1357_[1-2] were 
also amplified in diploid and tetraploid A. thaliana yielding bands with the same sizes in all the genotypes. Amplification with primers for the segment
Romania_aa1357_[2] revealed one band with $~ 300$ bp in both diploid and tetraploid $A$. thaliana and $A$. suecica but absent in $A$. arenosa.

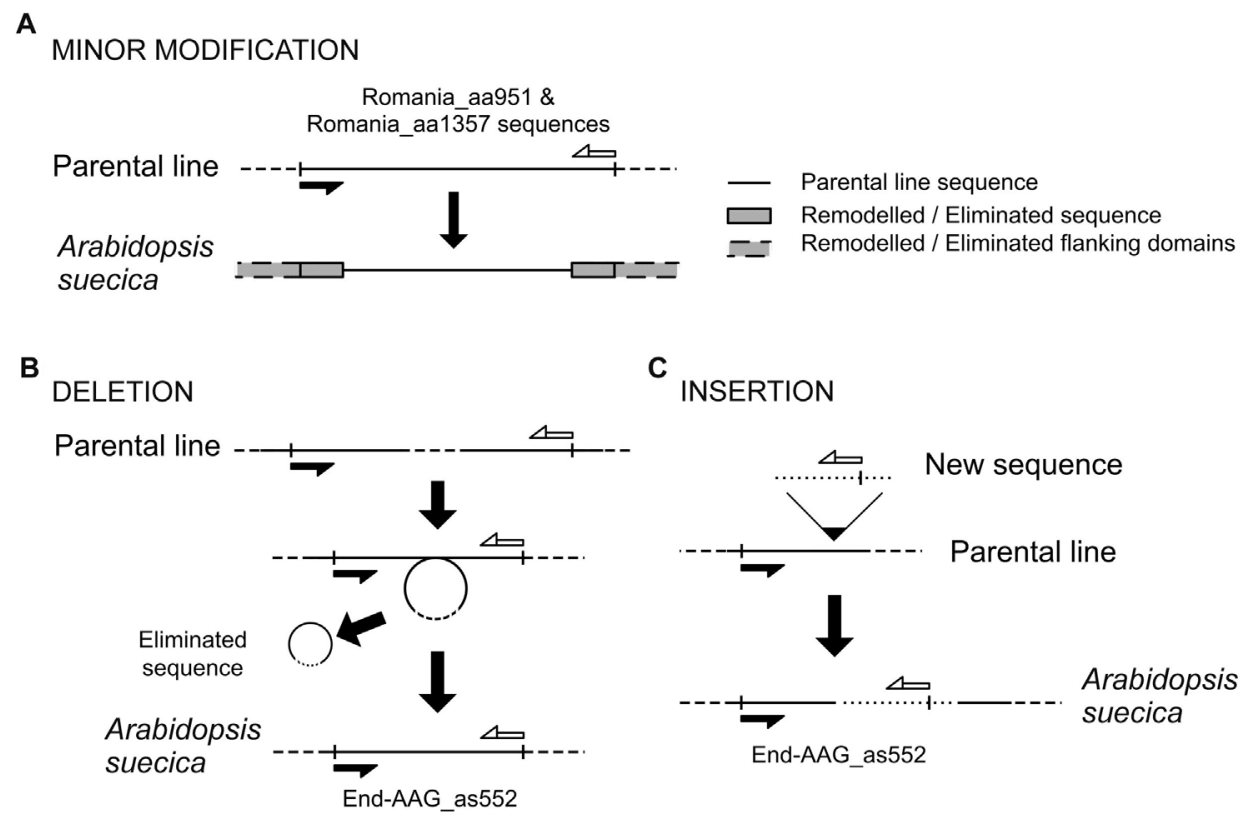

Fig. 4. $A$ - Schematic representation of IRAP RomaniaT5 A. arenosa origin sequences (Romania_aa951 and Romania_aa1357). Although original IRAP bands were absent in A. suecica, their internal fragments were detected in the allopolyploid genotype revealing that polyploidization probably induced changes restricted to flanking domains of primer annealing sites. $B$ and $C$ - Possible rearrangements induced by polyploidization in the origin of a novel REMAP Endovir1-1/(AAG)6C sequence (End-AAG_as552) detected exclusively in $A$. suecica $(B)$. It illustrates a deletion in one parental genome that allows the amplification of a novel PCR product; in $C$, it is illustrated the insertion of a sequence containing a primer annealing site into a new location near a second primer annealing site originating a new PCR product.

\section{Discussion}

The role of retrotransposon and microsatellite associated sequences in polyploid genome adjustment is hereby expressly corroborated through REMAP/IRAP evaluation of Arabidopsis auto- and allo-polyploid synthetic lines. The banding profiles of the $A$. thaliana autopolyploid line and its diploid progenitor revealed an overall genomic stability since all bands from the diploid line were observed in the autotetraploid one. Lack of parental sequence alterations was previously described in the A. thaliana autopolyploid through AFLP analysis (Ozkan et al. 2006). The undetection of band loss in the tetraploid A. thaliana line was expected considering the presence of multiple identical sequences due to its autopolyploid nature probably responsible for the maintenance of the described undistinguishable banding profiles. In fact, in the autotetraploid $A$. thaliana line, the only genomic alteration observed was a novel band detected with IRAP RomaniaT5. Conversely, in the A. suecica synthetic allopolyploid, an extensive rate of genome remodelling was observed corresponding to a major parental band elimination $(\sim 37 \%)$ and a minor appearance of novel bands $(4.6 \%)$. Sequence elimination/modification revealed by band loss was commonly detected in almost all allopolyploid species previously studied, such as triticale, Triticum, Brassica, Nicotiana, and Spartina (Song et al. 1995, Shaked et al. 2001, Kashkush et al. 2002, Ma et al. 2004, Dong et al. 2005, Ma and Gustafson 2006, Bento et al. 2008, Eilam et al. 2008, Petit et al. 2010). However, the present results unravelled in $A$. suecica through the IRAP/REMAP methodologies do not corroborate the preferential loss of bands from the larger genome parental species as observed using similar methodologies in allopolyploid systems with large genomes like wheat, triticale (Bento et al. 2010), and Cucumis $\times$ hytivus (Jiang et al. 2009). In triticale, the proportion of rye/wheat origin band loss $(20 / 5=4)$ (Bento et al. 2008) is considerably higher than the respective parental genome size ratio (8 600/5 $648 \mathrm{Mbp}=1.52$ ) (Bennett and Leitch 2010). Conversely, differences detected in the frequencies of $A$. arenosa origin and A. thaliana origin band losses $(24 / 36 \cong 67 \%$ and $12 / 36 \cong 33 \%$, respectively) in $A$. suecica $(24 / 12=2)$ were 
equivalent to the parental genome size proportion $(196 / 125 \mathrm{Mbp}=1.57)$ [196 bp - A. arenosa (Bennett and Leitch 2010); $125 \mathrm{bp}-A$. thaliana (AGI 2000).

The high parental band loss here detected (37 \%) markedly contrasts with the extremely low frequency of genomic modifications $(2.3 \%)$ detected in $A$. suecica when compared with the parental lines using AFLP (Madlung et al. 2005). The AFLP detects changes in restriction fragments occurring widely in all genome fractions and also detects epigenetic genome restructuring events if cytosine methylation sensitive restriction enzymes are used like in works previously referred (Madlung et al. 2005, Ozkan et al. 2006). Since the REMAP/IRAP methodologies are focused particularly on genome repetitive regions enriched in retrotransposons and/or microsatellites (Kalendar et al. 1999), the modifications now unravelled reinforces the idea that those repetitive sequences are preferentially altered by polyploidization as recently suggested (Bento et al. 2013). It would be tempting to address the involvement of those particularly labile sequences in natural $\mathrm{A}$. suecica genome adjustment. In fact, major restructuring events involving rDNA loci were similarly detected in both natural and synthetic $A$. suecica lines (Pontes et al. 2004). However, since the exact parental lines of the natural A. suecica lines are virtually unknown, it is impossible to accurately evaluate that interesting issue using molecular tools like IRAP and REMAP.

The BLASTN analysis through search in the NCBI, TAIR, and PlantSat databases of $A$. suecica remodelled bands revealed a high degree of similarity with coding sequences (six of eight bands sequenced, Table 1 Suppl.). From them, the analysis of all three remodelled bands detected through REMAP Endovir1-1/(AAG)6C unravelled sequences flanking microsatellites. This finding corroborates the idea that although microsatellites are present in both non-coding and coding regions, their frequency is higher in transcribed regions (RakoczyTrojanowska and Bolibok 2004). Moreover, this result clearly emphasises the role of microsatellite sequences in allopolyploid genome dynamics as recently proposed by Shi et al. (2013). The remaining restructured sequences detected through IRAP analysis, presenting a high similarity with $A$. thaliana coding sequences, suggest that although retrotransposons in Arabidopsis tend to be preferentially clustered within pericentromeric heterochromatin regions (Peterson-Burch et al. 2004), polyploidy induced genome adjustment seems to affect mainly LTR flanking sequences intermingled with generich domains. Despite the contrasting dimensions of repetitive sequence fractions in a plant with a large genomes like wheat (70 - $80 \%$ ) (Dvořák 2009) and with a small genome like $A$. thaliana ( $14 \%$ ) (AGI 2000) the present work ultimately shows that the levels of restructuring events targeting repetitive sequences of neighbouring regions are similar in allopolyploid species with both large (Bento et al. 2008) and small genome.

The amplification of internal segments of $A$. suecica remodelled sequences allowed a deeper understanding of major rearrangement occurrences. The internal amplifycation of the Romania_aa951 and Romania_aa1357 retrotransposon-related sequences in A. suecica suggests that the absence of parental bands in the allopolyploid might result from rearrangements confined to primer annealing site regions (Fig. 4A). Contrastingly, the amplification of the End-AAG as552 allopolyploid novel band using the primers targeting a domain with no significant homology with any known sequence corroborates the novelty of this restructured band since no amplification was detected in both the parental lines (Fig. $3 A$ ). The origin of such a new band in $A$. suecica could result either from the deletion of an intercalary sequence or from an insertion between the primer regions allowing the emergence of a new PCR amplicon (Fig. $4 B, C)$. Likewise, allopolyploid genome remodelling events now unravelled may be explained by a high rate of homoeologous pairing and intergenomic exchange recently reported to occur during meiosis of equivalent $A$. suecica neoallopolyploid lines (Henry et al. 2014).

Conclusively, the IRAP/REMAP comparative analysis performed in Arabidopsis auto- and allopolyploids clearly revealed distinct outcomes in newly formed polyploids. The overall genomic stability detected in the autopolyploid $A$. thaliana line, confirming previously described results using different techniques (Ozkan et al. 2006), contrasts with high parental genome remodelling in synthetic $A$. suecica involving repetitive and coding sequences. Furthermore, a deeper examination of parental lost sequences revealed that parental band loss mainly involved punctual changes on primers annealing sites in accordance with previous studies on allopolyploids with large genomes (Bento et al. 2010). However, contrary to allopolyploids with large genomes like triticale (Bento et al. 2008), a preferential modification of a larger parental genome was not observed revealing distinct parental genome behaviours in allopolyploid species with small genomes. The high parental band loss here reported suggest a major role of microsatellites and retrotransposons-related sequences in remodelling routes necessary to the establishment of new fertile polyploids. Moreover, repetitive sequence rearrangements may be highly involved in stable genetic changes needed to resolve parental genome conflicts that Comai et al. (2000) suggested to occur following the phenotypic instabilities and gene silencing events observed in the first generations of the same $A$. suecica synthetic line. 


\section{References}

AGI (The Arabidopsis Genome Initiative): Analysis of the genome sequence of the flowering plant Arabidopsis thaliana. - Nature 408: 796-815, 2000.

Ainouche, M.L.: Plant polyploidy. - New Phytol. 186 (Special Issue): 1-261, 2010.

Beaulieu, J., Jean, M., Belzile, F.: The allotetraploid Arabidopsis thaliana-Arabidopsis lyrata subsp. petraea as an alternative model system for the study of polyploidy in plants. - Mol. Genet. Genomics. 281: 421-435, 2009.

Bennett, M.D., Leitch, I.J.: Plant DNA C-values Database Release 6.0, Dec. 2012. http://data.kew.org/cvalues.

Bento, M., Gustafson, P., Viegas, W., Silva, M.: Genome merger: from sequence rearrangements in triticale to their elimination in wheat-rye addition lines. - Theor. appl. Genet. 121: 489-497, 2010.

Bento, M., Pereira, S., Rocheta, M., Gustafson, P., Viegas, W., Silva, M.: Polyploidization as a retraction force in plant genome evolution: sequence rearrangements in triticale. PLoS ONE 3: e1402, 2008.

Bento, M., Tomas, D., Viegas, W., Silva, M.: Retrotransposons represent the most labile fraction for genomic rearrangements in polyploid plant species. - Cytogenet. Genome Res. 140: 286-294, 2013.

Bomblies, K., Madlung, A.: Polyploidy in the Arabidopsis genus. - Chromosome Res. 22: 117-134, 2014.

Brenchley, R., Spannagl, M., Pfeifer, M., Barker, G.L., D'Amore, R., Allen, A.M., McKenzie, N., Kramer, M., Kerhornou, A., Bolser, D., Kay, S., Waite, D., Trick, M., Bancroft, I., Gu, Y., Huo, N., Luo, M.C., Sehgal, S., Gill, B., Kianian, S., Anderson, O., Kersey, P., Dvorak, J., McCombie, W.R., Hall, A., Mayer, K.F., Edwards, K.J., Bevan, M.W., Hall, N.: Analysis of the bread wheat genome using whole-genome shotgun sequencing. - Nature 491: 705-710, 2012 .

Comai, L., Tyagi, A.P., Winter, K., Holmes-Davis, R, Reynolds, S.H., Stevens, Y., Byers, B.: Phenotypic instability and rapid gene silencing in newly formed Arabidopsis allotetraploids. - Plant Cell 12: 1551-1567, 2000.

Dong, Y.Z., Liu, Z.L., Shan, X.H., Qiu, T., He, M.Y., Liu, B.: Allopolyploidy in wheat induces rapid and heritable alterations in DNA methylation patterns of cellular genes and mobile elements. - Russ. J. Genet. 41: 890-896, 2005.

Dvořák, J.: Triticeae genome structure and evolution. - In: Muehlbauer, G.J., Feuillet, C. (ed.): Genetics and Genomics of the Triticeae. Pp. 685-711. Springer, New York 2009.

Eilam, T., Anikster, Y., Millet, E., Manisterski, J., Feldman, M.: Nuclear DNA amount and genome downsizing in natural and synthetic allopolyploids of the genera Aegilops and Triticum. - Genome 51: 616-627, 2008.

Feldman, M., Levy, A.A.: Genome evolution in allopolyploid wheat-a revolutionary reprogramming followed by gradual changes. - J. Genet. Genomics 36: 511-518, 2009.

Ferreira-de-Carvalho, J., Chelaifa, H., Boutte, J., Poulain, J., Couloux, A., Wincker, P., Bellec, A., Fourment, J., Berge`s, H., Salmon, A., Ainouche, M.: Exploring the genome of the salt-marsh Spartina maritima (Poaceae, Chloridoideae) through BAC end sequence analysis. - Plant mol. Biol. 83: 591-606, 2013.

Hazzouri, R.M., Mohajer, A., Dejak, S.I., Otto, S.P., Wright, S.I.: Contrasting patterns of transposable-element insertion polymorphism and nucleotide diversity in autotetraploid and allotetraploid Arabidopsis species. - Genetics 179: 581-592, 2008.

Henry, I.M., Dilkes, B.P., Tyagi, A., Gao, J., Christensen, B., Comai, L.: The BOY NAMED SUE quantitative trait locus confers increased meiotic stability to an adapted natural allopolyploid of Arabidopsis. - Plant Cell. 26: 181-194, 2014.

Huang, S., Li, R., Zhang, Z., Li, L., Gu, X., Fan, W., Lucas, W.J., Wang, X., Xie, B., Ni, P., Ren, Y., Zhu, H., Li, J., Lin, K., Jin, W., Fei, Z., Li, G., Staub, J., Kilian, A., Van der Vossen, E.A., Wu, Y., Guo, J., He, J, Jia, Z., Ren, Y., Tian, G., Lu, Y., Ruan, J., Qian, W., Wang, M., Huang, Q., Li, B., Xuan, Z., Cao, J., Asan, Wu, Z., Zhang, J., Cai, Q., Bai, Y., Zhao, B., Han, Y., Li, Y., Li, X., Wang, S., Shi, Q., Liu, S., Cho, W.K., Kim, J.Y., Xu, Y., Heller-Uszynska, K., Miao, H., Cheng, Z., Zhang, S., Wu, J., Yang, Y., Kang, H, Li, M., Liang, H., Ren, X., Shi, Z., Wen, M., Jian, M., Yang, H., Zhang, G., Yang, Z., Chen, R., Liu, S., Li, J., Ma, L., Liu, H., Zhou, Y., Zhao, J., Fang, X., Li, G., Fang, L., Li, Y., Liu, D., Zheng, H., Zhang, Y., Qin, N., Li, Z., Yang, G., Yang, S., Bolund, L., Kristiansen, K., Zheng, H., Li, S., Zhang, X., Yang, H., Wang, J., Sun, R., Zhang, B., Jiang, S., Wang, J., Du, Y., Li, S.: The genome of the cucumber, Cucumis sativus L. - Nat. Genet. 41: 1275-1281, 2009.

Jiang, B., Lou, Q., Wu, Z., Zhang, W., Wang, D., Mbira, K.G., Weng, Y., Chen, J.: Retrotransposon- and microsatellite sequence-associated genomic changes in early generations of a newly synthesized allotetraploid Cucumis $\times$ hytivus Chen \& Kirkbride. - Plant mol. Biol. 77: 225-233, 2009.

Jiao, Y.N., Wickett, N.J., Ayyampalayam, S., Chanderbali, A.S., Landherr, L., Ralph, P.E., Tomsho, L.P., Hu, Y., Liang, H.Y., Soltis, P.S., Soltis, D.E., Clifton, S.W., Schlarbaum, S.E., Schuster, S.C., Ma, H., Leebens-Mack, J., De Pamphilis, C.W.: Ancestral polyploidy in seed plants and angiosperms. - Nature 473: 97-100, 2011.

Jones, R.N., Hegarty, M.: Order out of chaos in the hybrid plant nucleus. - Cytogenet. Genome Res. 126: 376-389, 2009.

Kalendar. R., Grob, T., Regina, M., Suoniemi, A., Schulman, A.: IRAP and REMAP: two new retrotransposon-based DNA fingerprinting techniques. - Theor. appl. Genet. 98: 704-711, 1999

Kalendar, R., Schulman, A.H.: IRAP and REMAP for retrotransposon-based genotyping and fingerprinting. - Nat. Protocols 1: 2478-2484, 2006.

Kashkush, K., Feldman, M., Levy, A.A.: Gene loss, silencing and activation in a newly synthesized wheat allotetraploid. Genetics 160: 1651-1659, 2002.

Ma, X.F., Fang, P., Gustafson, J.P.: Polyploidization-induced genome variation in triticale. - Genome 47: 839-848, 2004.

Ma, X.F., Gustafson, J.P.: Timing and rate of genome variation in triticale following allopolyploidization. - Genome 49: 950-958, 2006.

Ma, X.F., Gustafson, J.P.: Allopolyploidization-accommodated genomic sequence changes in triticale. - Ann. Bot. 101: 825-832, 2008

Madlung, A., Tyagi, A.P., Watson, B., Jiang, H.M., Kagochi, T., Doerge, R.W., Martienssen, R., Comai, L.: Genomic changes in synthetic Arabidopsis polyploids. - Plant J. 41: 221-230, 2005.

Ozkan, H., Tuna, M., Galbrainth, D.W.: No DNA loss in 
autotetraploid of Arabidopsis thaliana. - Plant Breed. 125: 288-291, 2006.

Parisod, C., Salmon, A., Zerjal, T., Tenaillon, M., Grandbastien, M.A., Ainouche, M.: Rapid structural and epigenetic reorganization near transposable elements in hybrid and allopolyploid genomes in Spartina. - New Phytol. 184: 1003-1015, 2009.

Pecinka, A., Fang, W., Rehmsmeier, M., Levy, A.A., Scheid, O.M.: Polyploidization increases meiotic recombination frequency in Arabidopsis. - BMC Biol. 9: 24, 2011.

Peterson-Burch, B.D., Nettleton, D., Voytas, D.F.: Genomic neighborhoods for Arabidopsis retrotransposons: a role for targeted integration in the distribution of the Metaviridae. Genome Biol. 5: R78, 2004.

Petit, M., Guidat, C., Daniel, J., Denis, E., Montoriol, E., Bui, Q.T., Lim, K.Y., Kovarik, A., Leitch, A.R., Grandbastien, M.A., Mhiri, C.: Mobilization of retrotransposons in synthetic allotetraploid tobacco. - New Phytol. 186: 135147, 2010.

Pontes, O., Neves, N., Silva, M., Lewis, M.S., Madlung, A., Comai, L., Viegas, W., Pikaard, C.S.: Chromosomal locus rearrangements are a rapid response to formation of the allotetraploid Arabidopsis suecica genome. - PNAS 101:18240-18245, 2004.

Rakoczy-Trojanowska, M., Bolibok, H.: Characteristics and a comparison of three classes of microsatellite-based markers and their application in plants. - Cell. mol. Biol. Lett. 9: 221-238, 2004.

Renny-Byfield, S., Kovarik, A., Kelly, L.J., Macas, J., Novak, P., Chase, M.W., Nichols, R.A., Pancholi, M.R., Grandbastien, M.A., Leitch, A.R.: Diploidization and genome size change in allopolyploids is associated with differential dynamics of low- and high-copy sequences. Plant J. 74: 829-839, 2013.

Rocheta, M., Cordeiro, J., Oliveira, M., Miguel, C.: PpRT1: the first complete gypsy-like retrotransposon isolated in Pinus pinaster. - Planta 225: 551-562, 2007.

Saghai-Maroof, M.A., Soliman, K.M., Jorgensen, R.A., Allard, R.W.: Ribosomal DNA spacer-length polymorphisms in barley - Mendelian inheritance, chromosomal location, and population-dynamics. - PNAS 81: 8014-8018, 1984.

Santos, J.L., Alfaro, D., Sanchez-Moran, E., Armstrong, S.J., Franklin, F.C.H.: Partial diploidization of meiosis in autotetraploid Arabidopsis thaliana. - Genetics 165: 15331540, 2003.

Shaked, H., Kashkush, K., Ozkan, H., Feldman, M., Levy, A.A.: Sequence elimination and cytosine methylation are rapid and reproducible responses of the genome to wide hybridization and allopolyploidy in wheat. - Plant Cell 13: 1749-1759, 2001.

Shi, J.Q., Huang, S.M., Fu, D.H., Yu, J.Y., Wang, X.F., Hua, W., Liu, S.Y., Liu, G.H., Wang, H.Z.: Evolutionary dynamics of microsatellite distribution in plants: insight from the comparison of sequenced Brassica, Arabidopsis and other angiosperm species. - Plos One. 8: e59988, 2013.

Sierro, N., Battey, J.N., Ouadi, S., Bovet, L., Goepfert, S., Bakaher, N., Peitsch, M.C., Ivanov, N.V.: Reference genomes and transcriptomes of Nicotiana sylvestris and Nicotiana tomentosiformis. - Genome Biol. 14: R60, 2013.

Song, K.M., Lu, P., Tang, K.L., Osborn, T.C.: Rapid genome change in synthetic polyploids of Brassica and Its implications for polyploid evolution. - PNAS 92: 77197723, 1995.

Stöck, M., Lamatsch, D.K.: Trends in polyploidy research in animals and plants. - Cytogenet. Genome Res. 140: 2-4, 2013.

Yu, Z., Haberer, G., Matthes, M., Rattei, T., Mayer, K.F.X., Gierl, A., Torres-Ruiz, R.A.: Impact of natural genetic variation on the transcriptome of autotetraploid Arabidopsis thaliana. - PNAS 107: 17809-17814, 2010. 